\title{
SUBSPACE SUPERCYCLICITY OF THE TUPLES OF OPERATORS
}

\author{
B. Yousefi ${ }^{1 \S}$, A. Sharifi ${ }^{2}$ \\ ${ }^{1,2}$ Department of Mathematics \\ Payame Noor University \\ P.O. Box 19395-4697, Tehran, IRAN
}

\begin{abstract}
In this paper, we investigate subspace supercyclicity for tuples of operators and we give conditions under which a tuple of operators satisfying the subspace supercyclicity criterion.
\end{abstract}

AMS Subject Classification: 47B37, 47B33

Key Words: tuple of operators, subspace-supercyclicity, subspace-hypercyclicity, supercylicity criterion

\section{Introduction}

By an n-tuple of operators we mean a finite sequence of length $\mathrm{n}$ of commuting continuous linear operators on a Banach space $X$.

Definition 1.1. Let $\mathcal{T}=\left(T_{1}, T_{2}, \ldots, T_{n}\right)$ be an n-tuple of operators acting on a separable infinite dimensional Banach space $X$ over $\mathbb{C}$ and let $M$ be a nonzero subspace of $X$. We will let $\mathcal{F}=\left\{T_{1}{ }^{k_{1}} T_{2}{ }^{k_{2}} \ldots T_{n}{ }^{k_{n}}: k_{i} \geq 0, i=1, \ldots, n\right\}$ be the semigroup generated by $\mathcal{T}$. For $x \in X$, the orbit of $x$ under the tuple $\mathcal{T}$ is the set $\operatorname{Orb}(\mathcal{T}, x)=\{S x: S \in \mathcal{F}\}$. A vector $x$ is called a $M$-supercyclic (M-hypercyclic) vector for $\mathcal{T}$ if $\operatorname{COrb}(\mathcal{T}, x) \cap M(\operatorname{Orb}(\mathcal{T}, x) \cap M)$ is dense in $M$ and in this case the tuple $\mathcal{T}$ is called $M$-supercyclic ( $M$-hypercyclic). The

Received: January 6, 2017

Revised: January 31, 2017

Published: February 9, 2017

$\S_{\text {Correspondence author }}$ (c) 2017 Academic Publications, Ltd. url: www.acadpubl.eu 
set of all $M$-supercyclic vectors of $\mathcal{T}$ is denoted by $S C(\mathcal{T}, M)$. Also, for all $k \geq 2$, by $\mathcal{T}_{d}^{(k)}$ we will refer to the set of all $k$ copies of an element of $\mathcal{F}$, i.e. $\mathcal{T}_{d}^{(k)}=\left\{S_{1} \oplus \ldots \oplus S_{k}: S_{1}=\ldots=S_{k} \in \mathcal{F}\right\}$. We say that $\mathcal{T}_{d}^{(k)}$ is subspacesupercyclic, with respect to $M$, provided there exist $x_{1}, \ldots, x_{k} \in X$ such that $\mathbf{C}\left\{W\left(x_{1} \oplus \ldots \oplus x_{k}\right): W \in \mathcal{T}_{d}^{(k)}\right\} \cap M$ is dense in the $k$ copies of $M, M \oplus \ldots \oplus M$.

Suprisingly, there are something that does not happen for single operators. For example, supercyclic tuples can arise in finite dimensional, and there are operators that have somewhere dense orbits that are not everywhere dense. Also, we note that there are subspace-supercyclic operators that are not supercyclic. For some topics we refer to [1]-[2].

\section{Main Results}

In this section, we investigate an equivalent condition for subspace-supercyclicity criterion for tuples of operators.

Theorem 2.1. Let $M$ be a nonzero subspace of separable infinite dimensional Banach space $X$ and $\mathcal{T}=\left(T_{1}, T_{2}, \ldots, T_{n}\right)$ be a tuple of operators $T_{1}, T_{2}$, $\ldots, T_{n}$. Then $\mathcal{T}$ is $M$-supercyclic if and only if for any two nonempty relatively open sets $U$ and $V$ in $M$, there exist $m_{i} \geq 1$ for $i=1, \ldots, n$ and and $\lambda \in \mathbb{C} \backslash\{0\}$ such that $\lambda T_{1}^{m_{1}} \ldots T_{n}^{m_{n}}(U) \cap V$ is nonempty and $T_{1}^{m_{1}} \ldots T_{n}^{m_{n}} M \subset M$.

Proof. Suppose that $x$ is a $M$-supercyclic vector for $\mathcal{T}$, hence $\operatorname{COrb}(\mathcal{T}, x) \cap$ $M$ is dense in $M$ and so there exist $\lambda_{1} \in \mathbf{C} \backslash\{0\}$ and $m_{i} \in \mathbb{N} ; i=1, \ldots, n$ such that $\lambda_{1} T_{1}^{m_{1}} \ldots T_{n}^{m_{n}} x \in U$. Let $y=\lambda_{1} T_{1}^{m_{1}} \ldots T_{n}^{m_{n}} x$. Since $\mathbf{C O r b}(\mathcal{T}, x) \cap$ $M$ is also dense in $M$, thus $\lambda T_{1}^{m_{1}} \ldots T_{n}^{m_{n}} y \in V$ for some $\lambda \in \mathbf{C} \backslash\{0\}$ and $m_{i} \in \mathbb{N} ; i=1, \ldots, n$, hence $\lambda T_{1}^{m_{1}} \ldots T_{n}^{m_{n}}(U) \cap V$ is nonempty. To show that $T_{1}^{m_{1}} T_{2}^{m_{2}} \ldots T_{n}^{m_{n}} M \subset M$, let $x \in M$ and let $W$ be a relatively open nonempty subset of $\lambda^{-1} T_{1}^{-m_{1}} \ldots T_{n}^{-m_{n}}(V) \cap U$ in $M$. Note that

$$
\lambda T_{1}^{m_{1}} T_{2}^{m_{2}} \ldots T_{n}^{m_{n}} W \subset V \cap \lambda T_{1}^{m_{1}} T_{2}^{m_{2}} \ldots T_{n}^{m_{n}} U \subset V \subset M .
$$

Hence $T_{1}^{m_{1}} T_{2}^{m_{2}} \ldots T_{n}^{m_{n}} W \subset M$. If $x_{0} \in W$, then there exists $r>0$ small enough such that $x_{0}+r x \in W$, since $W$ is relatively open. Thus

$$
T_{1}^{m_{1}} T_{2}^{m_{2}} \ldots T_{n}^{m_{n}}\left(x_{0}+r x\right)=T_{1}^{m_{1}} T_{2}^{m_{2}} \ldots T_{n}^{m_{n}} x_{0}+r T_{1}^{m_{1}} T_{2}^{m_{2}} \ldots T_{n}^{m_{n}} x \in M,
$$

which implies that $T_{1}^{m_{1}} T_{2}^{m_{2}} \ldots T_{n}^{m_{n}} x \in M$. Thus $T_{1}^{m_{1}} T_{2}^{m_{2}} \ldots T_{n}^{m_{n}} M \subset M$. For the converse, fix an enumeration $\left\{B_{n}: n \in \mathbb{N}\right\}$ of relatively open balls in $M$ with 
rational radii, and centers in a countable dense subset of $M$. By the continuity of the operators $T_{1}, T_{2}, \ldots, T_{n}$, the sets

$$
G_{k}=\bigcup\left\{\lambda T_{1}^{-m_{1}} \ldots T_{n}^{-m_{n}}\left(B_{k}\right) \cap M: m_{i} \geq 0 ; i=1, \ldots, n, \lambda \in \mathbb{C}\right\}
$$

are relatively open in $M$. Since for any two nonempty relatively open sets $U$ and $V$ in $M$, there exist $m_{i} \geq 1 ; i=1, \ldots, n$, and $\lambda \in \mathbf{C} \backslash\{0\}$ such that $\lambda T_{1}^{m_{1}} \ldots T_{n}^{m_{n}}(U) \cap V$ contains a relatively open nonempty subset of $M$, thus one can see that $S C(\mathcal{T})$ is exactly equal to $\bigcap\left\{G_{k}: k \in \mathbb{N}\right\}$ that is dense in $M$.

Theorem 2.2. (Subspace-Supercyclicity Criterion for Tuples) Let $\mathcal{T}=$ $\left(T_{1}, T_{2}, \ldots, T_{n}\right)$ be a tuple of continuous operators acting on a separable infinite dimensional Banach space $X$ and $M$ be a nonzero closed subspace of $X$. Suppose that there exist two dense subsets $Y$ and $Z$ in $M$, and strictly increasing sequences of positive integers $\left\{m_{j(i)}\right\}_{j}$ for $i=1, \ldots, n$, and a sequence of mappings $S_{j}: Z \rightarrow M$ such that:

1. For every $z \in Z, T_{1}^{m_{j(1)}} \ldots T_{n}^{m_{j(n)}} S_{j} z \rightarrow z$,

2. $\left\|T_{1}^{m_{j(1)}} \ldots T_{n}^{m_{j(n)}} y\right\|\left\|S_{j} z\right\| \rightarrow 0$ for all $y \in Y$ and all $z \in Z$,

3. $M$ is invariant subspace for $T_{1}^{m_{j(1)}} \ldots T_{n}^{m_{j(n)}}$ for all $j$.

Then $\mathcal{T}$ is subspace-supercyclic with respect to $M$.

Proof. Let $U$ and $V$ be two nonempty relatively open subsets of $M$. Choose $v \in Y \cap V$ and $u \in Z \cap U$. Since $U$ and $V$ are relatively open, there exists $\epsilon>0$ such that $B(v, \epsilon) \cap M \subset V$ and $B(u, \epsilon) \cap M \subset U$. By the hypothesis we have: (i). $T_{1}^{m_{j(1)}} \ldots T_{n}^{m_{j(n)}} S_{j} u \rightarrow u, \quad$ (ii). $\left\|T_{1}^{m_{j(1)}} \ldots T_{n}^{m_{j(n)}} v\right\|\left\|S_{j} u\right\| \rightarrow 0$, (iii). $M$ is invariant subspace for $T_{1}^{m_{j(1)}} \ldots T_{n}^{m_{j(n)}}$ for all $j$.

Hence for large $j$, we can find $\lambda_{j} \in \mathbf{C} \backslash\{0\}$ such that

$$
\left\|\lambda_{j} T_{1}^{m_{j(1)}} \ldots T_{n}^{m_{j(n)}} v\right\|<\epsilon / 2,\left\|\lambda_{j}^{-1} S_{j} u\right\|<\epsilon,\left\|T_{1}^{m_{j(1)}} \ldots T_{n}^{m_{j(n)}} S_{j} u-u\right\|<\epsilon / 2 .
$$

Note that since $v \in M$ and $S_{j} u \in M$, we have $v+\lambda_{j}^{-1} S_{j} u \in M$ and $\|(v+$ $\left.\lambda_{j}^{-1} S_{j} u\right)-v\|=\| \lambda_{j}^{-1} S_{j} u \|<\epsilon$. Hence $v+S_{j} u \in V$. Also we note that $T_{1}^{m_{j(1)}} \ldots T_{n}^{m_{j(n)}}\left(v+\lambda_{j}^{-1} S_{j} u\right) \in M$ and $\left\|\lambda_{j} T_{1}^{m_{j(1)}} \ldots T_{n}^{m_{j(n)}}\left(v+\lambda_{j}^{-1} S_{j} u\right)-u\right\|<\epsilon$. Thus $\lambda_{j} T_{1}^{m_{j(1)}} \ldots T_{n}^{m_{j(n)}}\left(v+\lambda_{j}^{-1} S_{j} u\right) \in B(u, \epsilon) \cap M$ and so $\lambda_{j} T_{1}^{m_{j(1)}} \ldots T_{n}^{m_{j(n)}}(v+$ $\left.\lambda_{j}^{-1} S_{j} u\right) \in U$. Hence $v+\lambda_{j}^{-1} S_{j} u \in \lambda_{j}^{-1} T_{1}^{-m_{j(1)}} \ldots T_{n}^{-m_{j(n)}}(U) \cap V$ and so by Theorem 2.1, $\mathcal{T}$ is subspace-supercyclic with respect to $M$. 
Theorem 2.3. Let $M$ be a nonzero subspace of a separable infinite dimensional Banach space $X$ and $\mathcal{T}=\left(T_{1}, T_{2}, \ldots, T_{n}\right)$ be a tuple of operators $T_{1}, T_{2}, \ldots, T_{n}$. If $\mathcal{T}_{d}^{(2)}$ is $M \oplus M$-supercyclic, then $\mathcal{T}$ satisfies the SubspaceSupercyclicity Criterion with respect to $M$.

Proof. Suppose that $\mathcal{T}_{d}^{(2)}$ is $M \oplus M$-supercylcic and let $(x, y)$ be a $M \oplus M$ supercyclic vector for $\mathcal{T}_{d}^{(2)}$. In particular $x$ and $y$ are supercyclic vectors for $\mathcal{T}$. For all $k \in \mathbb{N}$, put $U_{k}=B\left(0, \frac{1}{k}\right) \cap M$. Then there exist $\left\{m_{j_{i}}\right\}_{j} \subset \mathbb{N}$ for $i=1, \ldots, n$ and $\lambda_{j} \in \mathbf{C}$ such that

$$
\begin{gathered}
\lambda_{j}\left(T_{1}^{m_{j_{1}}} T_{2}^{m_{j_{2}}} \ldots T_{n}^{m_{j_{n}}} \oplus T_{1}^{m_{j_{1}}} T_{2}^{m_{j_{2}}} \ldots T_{n}^{m_{j_{n}}}\right)(x, y) \in U_{j} \oplus\left(x+U_{j}\right), \\
T_{1}^{m_{j_{1}}} T_{2}^{m_{j_{2}}} \ldots T_{n}^{m_{j_{n}}} \oplus T_{1}^{m_{j_{1}}} T_{2}^{m_{j_{2}}} \ldots T_{n}^{m_{j_{n}}} M \oplus M \subset M \oplus M .
\end{gathered}
$$

Therefore, $\lambda_{j} T_{1}^{m_{j_{1}}} T_{2}^{m_{j_{2}}} \ldots T_{n}^{m_{j_{n}}} x \in U_{j}, \quad \lambda_{j} T_{1}^{m_{j_{1}}} T_{2}^{m_{j_{2}}} \ldots T_{n}^{m_{j_{n}}} y \in x+U_{j}$, and $T_{1}^{m_{j_{1}}} T_{2}^{m_{j_{2}}} \ldots T_{n}^{m_{j_{n}}} M \subset M$ for all $j \in \mathbb{N}$. This implies that

$$
\lambda_{j} T_{1}^{m_{j_{1}}} T_{2}^{m_{j_{2}}} \ldots T_{n}^{m_{j_{n}}} x \rightarrow 0, \lambda_{j} T_{1}^{m_{j_{1}}} T_{2}^{m_{j_{2}}} \ldots T_{n}^{m_{j_{n}}} y \rightarrow x .
$$

Let $Y=Z=\operatorname{COrb}(\mathcal{T}, x)$ which is dense in $M$. Also for all $j \in \mathbb{N}, \lambda \in \mathbf{C}$ and $k_{r_{i}} \in \mathbb{N}$ forall $i=1, \ldots, n$, define $S_{j}\left(\lambda T_{1}^{k_{r_{1}}} \ldots T_{n}^{k_{r_{n}}} x\right)=\lambda \lambda_{j} T_{1}^{k_{r_{1}}} \ldots T_{n}^{k_{r_{n}}} y$. Note that $T_{1}^{m_{j_{1}}} \ldots T_{n}^{m_{j_{n}}} S_{j}\left(\lambda T_{1}^{k_{r_{1}}} \ldots T_{n}^{k_{r_{n}}} x\right)=\lambda T_{1}^{k_{r_{1}}} \ldots T_{n}^{k_{r_{n}}}\left(\lambda_{j} T_{1}^{m_{j_{1}}} \ldots T_{n}^{m_{j_{n}}} y\right)$ which tends to $\lambda T_{1}^{k_{r_{1}}} T_{2}^{k_{r_{2}}} \ldots T_{n}^{k_{r_{n}}} x$ as $j \rightarrow \infty$. So $T_{1}^{m_{j_{1}}} T_{2}^{m_{j_{2}}} \ldots T_{n}^{m_{j_{n}}} S_{j} z \rightarrow z$ for all $z \in Z$. Also for all $\lambda, w \in \mathbb{C}$ and $t_{s_{i}}, k_{r_{i}} \in \mathbb{N}$ for $i=1, \ldots, n$, clearly we can see that $\left\|T_{1}^{m_{j_{1}}} T_{2}^{m_{j_{2}}} \ldots T_{n}^{m_{j_{n}}}\left(\lambda T_{1}^{t_{s_{1}}} T_{2}^{t_{s_{2}}} \ldots T_{n}^{t_{s_{n}}} x\right)\right\|\left\|S_{j}\left(w T_{1}^{k_{r_{1}}} T_{2}^{k_{r_{2}}} \ldots T_{n}^{k_{r_{n}}} x\right)\right\| \rightarrow 0$ as $j \rightarrow \infty$. Thus for all $y \in Y$ and $z \in Z$, we get $\left\|T_{1}^{m_{j_{1}}} T_{2}^{m_{j_{2}}} \ldots T_{n}^{m_{j_{n}}} y\right\|\left\|S_{j} z\right\| \rightarrow 0$ and so $\mathcal{T}$ satisfies the $M$-Supercyclicity Criterion.

\section{References}

[1] N.S. Feldman, Hypercyclic tuples of operators and somewhere dense orbits, J. Math. Appl., 346 (2008), 82-98.

[2] B.F. Madore and R.A. Martinez-Avendano, Subsapce hypercyclicity, Journal of Mathematical Analysis and Applications, 375, No. 2 (2011), 502-511. 